\title{
UNVEILING THE ORIGIN OF GRB 090709A: LACK OF PERIODICITY IN A REDDENED COSMOLOGICAL LONG-DURATION GAMMA-RAY BURST
}

\author{
S. B. Cenko ${ }^{1}$, N. R. Butler ${ }^{1,10}$, E. O. Ofer ${ }^{2,10}$, D. A. Perley ${ }^{1}$, A. N. Morgan ${ }^{1}$, D. A. Frail ${ }^{3}$, J. Gorosabel ${ }^{4}$, J. S. Bloom ${ }^{1}$, \\ A. J. Castro-Tirado ${ }^{4}$, J. Cepa ${ }^{5}$, P. Chandra ${ }^{6}$, A. De Ugarte Postigo ${ }^{7}$, A. V. Filippenko ${ }^{1}$, C. R. Klein ${ }^{1}$, S. R. Kulkarni ${ }^{2}$, \\ A. A. Miller ${ }^{1}$, P. E. Nugent ${ }^{8}$, AND D. L. StarR ${ }^{1,9}$ \\ ${ }^{1}$ Department of Astronomy, University of California, Berkeley, CA 94720-3411, USA \\ 2 Division of Physics, Mathematics, and Astronomy, California Institute of Technology, Pasadena, CA 91125, USA \\ ${ }^{3}$ National Radio Astronomy Observatory, 1003 Lopezville Road, Socorro, NM 87801, USA \\ ${ }^{4}$ Instituto de Astrofísica de Andalucía (IAA-CSIC), Apartado de Correos, 3.004, E-18.080 Granada, Spain \\ ${ }^{5}$ Instituto de Astrofísica de Canarias, 38205 La Laguna, Spain \\ ${ }^{6}$ Department of Physics, Royal Military College of Canada, Kingston, ON, Canada \\ ${ }^{7}$ INAF/Osservatorio Astronomico di Brera, via Bianchi 46, 23807 Merate, LC, Italy \\ ${ }^{8}$ Lawrence Berkeley National Laboratory, 1 Cyclotron Road, Berkeley, CA 94720, USA \\ ${ }^{9}$ Las Cumbres Observatory Global Telescope Network, Inc., 6740 Cortona Drive, Suite 102, Goleta, CA 93117, USA \\ Received 2009 November 16; accepted 2010 May 13; published 2010 June 10
}

\begin{abstract}
We present broadband (gamma-ray, X-ray, near-infrared, optical, and radio) observations of the Swift gamma-ray burst (GRB) 090709A and its afterglow in an effort to ascertain the origin of this high-energy transient. Previous analyses suggested that GRB 090709A exhibited quasi-periodic oscillations with a period of $8.06 \mathrm{~s}$, a trait unknown in long-duration GRBs but typical of flares from soft gamma-ray repeaters. When properly accounting for the underlying shape of the power-density spectrum of GRB 090709A, we find no conclusive $(>3 \sigma)$ evidence for the reported periodicity. In conjunction with the location of the transient (far from the Galactic plane and absent any nearby host galaxy in the local universe) and the evidence for extinction in excess of the Galactic value, we consider a magnetar origin relatively unlikely. A long-duration GRB, however, can account for the majority of the observed properties of this source. GRB 090709A is distinguished from other long-duration GRBs primarily by the large amount of obscuration from its host galaxy ( $A_{K \text {,obs }} \gtrsim 2 \mathrm{mag}$ ).
\end{abstract}

Key words: gamma-ray burst: general - stars: neutron

Online-only material: color figures

\section{INTRODUCTION}

A variety of astrophysical sources are capable of producing short, intense flashes of high-energy emission detectable by the current generation of gamma-ray satellites. These sources span an incredible range of the observable universe, from electrical discharges associated with thunderstorms on Earth (Fishman et al. 1994) to the deaths of the earliest known stars in the universe (Tanvir et al. 2009; Salvaterra et al. 2009).

Gamma-ray bursts (GRBs) are the most luminous class of these high-energy transients $\left(L_{\text {iso }} \approx 10^{50}-10^{52} \mathrm{erg} \mathrm{s}^{-1}\right)$. At least two distinct progenitor systems are thought to produce GRBs (Kouveliotou et al. 1993). It is now widely accepted that most GRBs with duration $t_{90} \gtrsim 2 \mathrm{~s}$ arise as a byproduct of the core collapse of massive stars (hereafter referred to as "longduration" GRBs; e.g., Woosley \& Bloom 2006, and references therein). The origin of "short-duration" GRBs is still a hotly debated topic. They likely arise from an older stellar population (e.g., Gehrels et al. 2005; Hjorth et al. 2005; Barthelmy et al. 2005b; Castro-Tirado et al. 2005; Berger et al. 2005; Bloom et al. 2006b; Gorosabel et al. 2006), possibly due to the merger of a neutron star-neutron star (NS-NS) or black hole-neutron star (BH-NS) binary (Eichler et al. 1989; Narayan et al. 1992).

Soft gamma-ray repeaters (SGRs), on the other hand, are distinguished from GRBs by repeated outbursts with an isotropic energy release of $E_{\gamma \text {,iso }} \lesssim 10^{46}$ erg (e.g., Woods \& Thompson 2006; Ofek 2007; Mereghetti 2008). The discovery of periodic

\footnotetext{
${ }^{10}$ Einstein Fellow.
}

oscillations from bright SGR flares (Mazets et al. 1979), along with the measurement of a spin-down in their periods (Kouveliotou et al. 1998), allows for an estimation of the magnetic field strength. Unlike typical rotation-powered radio pulsars, SGRs (as well as their counterparts discovered in quiescence, the anomalous X-ray pulsars, or AXPs) are likely powered by their intense magnetic fields $\left(B \gtrsim 10^{14} \mathrm{G}\right.$; Duncan \& Thompson 1992). Together, SGRs and AXPs are now thought to comprise a single class of young, highly magnetized NSs ("magnetars").

At least three discoveries have in recent years challenged this simple classification picture. First, both GRB 060614 and GRB 060505 had high-energy durations in excess of a few seconds-yet despite being quite nearby $(z \approx 0.1)$, neither exhibited evidence for an associated supernova to quite deep limits (Gehrels et al. 2006; Gal-Yam et al. 2006; Della Valle et al. 2006; Fynbo et al. 2006; Ofek et al. 2007). Second, many GRBs that would have been classified as having short duration $\left(t_{90} \lesssim 2 \mathrm{~s}\right.$ ) by the less sensitive BATSE satellite exhibit soft (and oftentimes faint) $\mathrm{X}$-ray tails extending hundreds of seconds in time when observed by Swift (e.g., Barthelmy et al. 2005b; Perley et al. 2009a) - perceived duration is, after all, at once a redshift-, sensitivity-, and bandpass-dependent quantity. Finally, the X-ray and optical afterglow light curves of GRB 070610 $\left(t_{90}=5 \mathrm{~s}\right)$ displayed dramatic flares on time scales as short as several seconds as late as days after the burst (Kasliwal et al. 2008; Stefanescu et al. 2008; Castro-Tirado et al. 2008). Though almost certainly a Galactic source, the nature of this variability is still poorly understood. 
Here we present observations of GRB 090709A, a highenergy transient whose classification as a traditional longduration GRB has been called into question. Markwardt et al. (2009) reported the detection of quasi-periodic oscillations (QPOs; period $P=8.06 \mathrm{~s}$ ) in the high-energy light curve, typical of the observed properties of SGRs and AXPs. However, unlike all currently known magnetars, the localization of GRB 090709A is inconsistent with both the Galactic plane and any nearby galaxy. Through a detailed analysis of the highenergy light curve, the broadband afterglow, and the immediate environment, we attempt to uncover the true origin of GRB 090709A.

Throughout this work we adopt a standard $\Lambda \mathrm{CDM}$ cosmology $\left(\Omega_{\Lambda}=0.73 ; \Omega_{m}=0.27 ; H_{0}=71 \mathrm{~km} \mathrm{~s}^{-1} \mathrm{Mpc}^{-1}\right)$. All quoted uncertainties are $1 \sigma$ (i.e., $68 \%$ ) confidence intervals unless otherwise stated. Spectral and temporal power-law indices are provided using the convention $f_{v} \propto t^{-\alpha} v^{-\beta}$ (Sari et al. 1998). UT dates are used throughout this work.

In the final stages of preparing this paper, De Luca et al. (2010) posted an analysis of X-ray (XMM-Newton and Swift-X-ray Telescope (XRT)) and gamma-ray (Swift-Burst Alert Telescope (BAT) and Integral-SPI/ACS) observations of GRB 090709A. These authors reach largely similar conclusions regarding the origin of GRB 090709A, although they favor a somewhat greater distance for the event. We attempt to highlight both the differences and similarities between our work in what follows.

\section{HIGH-ENERGY PROPERTIES}

GRB 090709A was detected by BAT (Barthelmy et al. 2005a) on board the Swift satellite (Gehrels et al. 2004) at 7:38:34 on 2009 July 9 ( $t_{0}$; Morris et al. 2009). In Figure 1, we plot the 15-350 keV BAT light curve, binned with $1 \mathrm{~s}$ time resolution, obtained following the prescription described by Butler et al. (2007). The emission is dominated by a broad peak beginning at $t_{0}$ and lasting approximately $100 \mathrm{~s}$. However, there is evidence for low-level variability well before the trigger $\left(t \approx t_{0}-70 \mathrm{~s}\right)$, and the light curve appears to rise again at $t \approx t_{0}+400 \mathrm{~s}$. Shortly thereafter the spacecraft slewed away due to an observing constraint (Sakamoto et al. 2009). Including data from $t_{0}-66 \mathrm{~s}$ to $t_{0}+509 \mathrm{~s}$, we measure $t_{90}=345 \pm 64 \mathrm{~s}$ $(15-350 \mathrm{keV})$. Over this interval we find that the spectrum is best fit by a power law with an exponential cutoff, with $\alpha=$ $1.06 \pm 0.14$ and $E_{\mathrm{p}}=299_{-101}^{+547} \mathrm{keV}\left(\chi^{2}=38.71\right.$ for 55 degrees of freedom, dof). The corresponding $15-350 \mathrm{keV}$ fluence is $f=4.59_{-0.26}^{+0.30} \times 10^{-5} \mathrm{erg} \mathrm{cm}^{-2}$, making GRB 090709A one of the brightest Swift events detected to date.

Superposed on the overall $\sim 100$ s duration rise and decline of the dominant emission component, the high-energy light curve exhibits relatively strong fluctuations. In the inset of Figure 1, we plot the residual emission after subtracting a smoothed version of the 15-350 keV light curve (boxcar binned by $10 \mathrm{~s}$ ).

Markwardt et al. (2009) first reported a search for a periodic signal from GRB 090709A, claiming a detection of an excess in the power density spectrum (PDS) at $P=8.06 \mathrm{~s}$. To estimate the significance of this excess, Markwardt et al. (2009) normalized the entire PDS by the noise in the observed frequency range $0.2-0.6 \mathrm{~Hz}$. After correcting for the number of frequency bins examined $(\sim 2000)$ and the estimated number of bursts for which such an analysis could be performed $(\sim 100)$, the authors concluded that the observed peak is highly significant (null probability $\sim 10^{-6}$ ). The detection of this apparent periodicity was subsequently confirmed with data from several additional

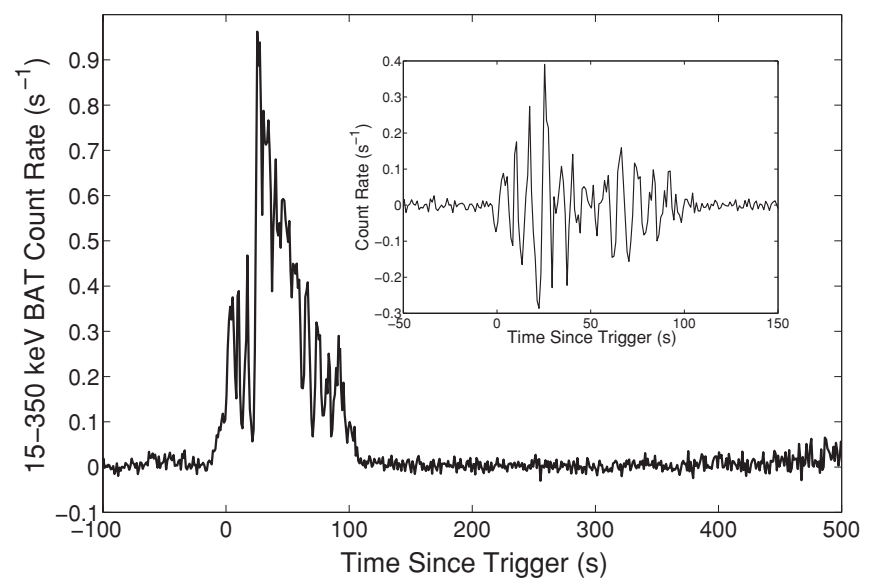

Figure 1. Swift-BAT 15-350 keV light curve of GRB 090709A, referenced to the time of the BAT trigger, 7:38:34 UT on 2009 July 9. The high-energy emission is dominated by a $\sim 100 \mathrm{~s}$ long peak beginning at $t_{0}$, although fainter emission is visible both before and after this episode. The inset shows the residual emission after subtracting a smoothed version of the light curve.

high-energy satellites (Golenetskii et al. 2009; Gotz et al. 2009; Ohno et al. 2009).

In the left panel of Figure 2, we plot the unnormalized PDS of GRB 090709A. The 15-350 keV light curve from $t_{0}$ to $t_{0}+100 \mathrm{~s}$ was binned with a time resolution of $10 \mathrm{~ms}$ and detrended by subtracting a smoothed version of the light curve (10 s boxcar). The peak noted by Markwardt et al. (2009) is clearly visible at a period of $8.1 \mathrm{~s}$. It is crucial to note, however, that the interpretation of the significance of this peak depends sensitively on the assumed noise properties and the underlying shape of the PDS. We therefore examine this issue here in greater detail.

The simplest strategy to infer the statistical significance of this feature is to assume that the PDS is dominated by white noise (i.e., independent of frequency). The significance (signalto-noise ratio, $\mathrm{S} / \mathrm{N}$ ) can then be estimated by normalizing the PDS by the observed scatter in a region devoid of features. Following Markwardt et al. (2009), we normalize the PDS of GRB 090709A with respect to the observed scatter in the range $P=3-6.5$ s (one of the noisier regions of the PDS). The result is shown as the dashed line in the right panel of Figure 2. After correcting for $\sim 10^{5}$ trials $(\sim 1000$ period bins using our $10 \mathrm{~ms}$ light curve and considering $0 \lesssim P \lesssim 10$ s for $\sim 100$ longduration GRBs), we find that the observed peak at $P=8.1 \mathrm{~s}$ is significant at the $12 \sigma$ level.

The PDSs of GRB prompt emission, however, are not featureless. Examining the left panel of Figure 2, three distinct regions can be defined. For the shortest periods $(P \lesssim 1 \mathrm{~s}$ ), the PDS is relatively flat and dominated by Poisson statistical fluctuations (white noise). For large periods ( $P \gtrsim 10 \mathrm{~s}$ ), the PDS is dominated by the total duration of the analyzed light curve (the window function, $\sim 100$ s for GRB 090709A) and the detrending algorithm ( $\sim 10$ s smoothing for GRB 090709A).

For intermediate periods $(1 \lesssim P \lesssim 10 \mathrm{~s})$, Beloborodov et al. (1998) have shown that the PDSs of the longest $\left(t_{90}>100 \mathrm{~s}\right)$ BATSE bursts are well fit by a power law with index $\alpha \approx 5 / 3$ (where power $\propto P^{\alpha}$ ). Since the average power-law index was found to be in close agreement with the Kolmogorov law, it has been suggested that the prompt emission may be related to turbulence in the outflow (Beloborodov et al. 2000). Subsequent analysis has indicated, however, that the exact value of the power-law slope can vary significantly from event to event and is determined predominantly by the shape and duration 

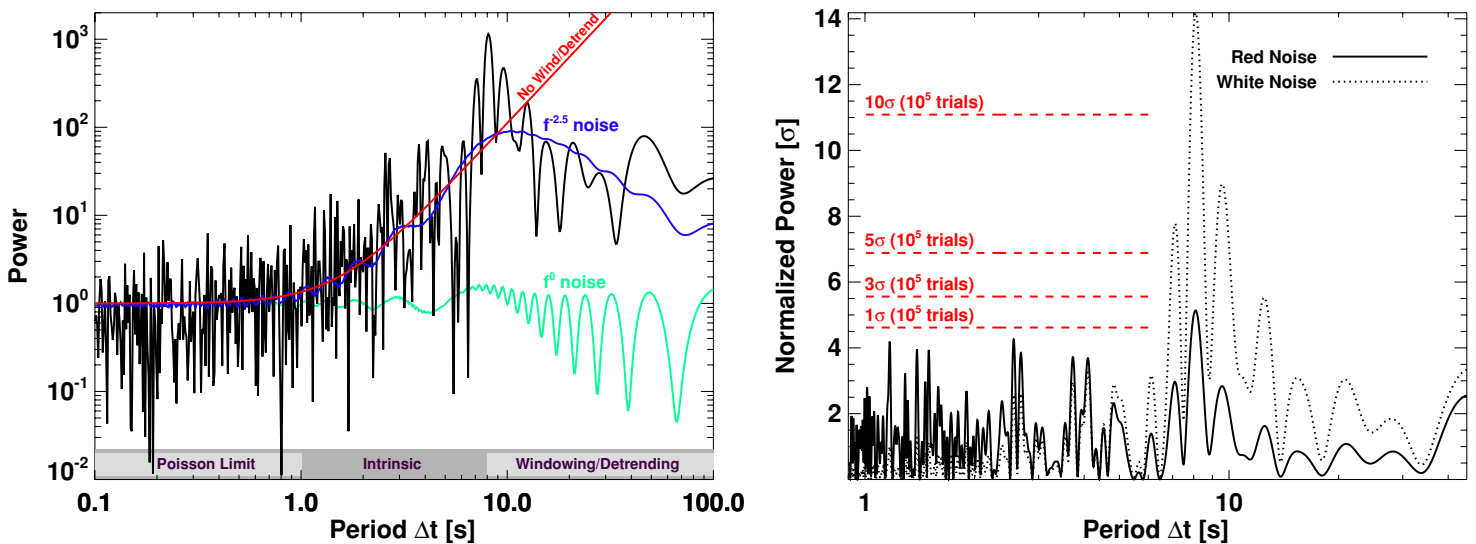

Figure 2. Left: unnormalized PDS of GRB 090709A. The PDS was constructed using the $15-350 \mathrm{keV}$ BAT light curve from $t_{0}$ to $t_{0}+100 \mathrm{~s}$. A smoothed (10 s boxcar) version of the light curve was first subtracted to remove the overall rise and decline trend. Three clear regions are visible. For $P \lesssim 1 \mathrm{~s}$, the PDS is dominated by Poisson statistics (i.e., white noise). For $P \gtrsim 10 \mathrm{~s}$, the effects of windowing and detrending dominate the error in the PDS. The power-law slope in the intermediate regime $(\alpha=2.5)$, intrinsic to the GRB prompt emission but simulating the effects of red noise, is clearly visible. The red line shows our model for the underlying shape of the PDS derived using a nonwindowed light curve absent detrending. Noise models assuming a flat PDS (green line, $f^{0}$ noise) and a power-law PDS (blue line, $f^{2.5}$ noise) are also shown. Right: normalized PDS of GRB 090709A. The dashed line assumes a flat underlying spectrum (white noise), while the solid line accounts for the intrinsic fluctuations (red noise). Significance intervals assuming $10^{5}$ trials are indicated by the horizontal dashed blue lines. The significance of the observed feature at $P=8.1$ s drops dramatically after accounting for the underlying power-law spectrum.

(A color version of this figure is available in the online journal.)

of individual pulse episodes within the GRB (Suzuki et al. 2002). PDS measurements of bright Swift events have been performed (e.g., GRB 080319B, Bloom et al. 2009) and show results consistent with those from BATSE.

Regardless of its origin, it is clear that we must include the underlying behavior of the PDS when evaluating the significance of individual features. The underlying slope is not "noise" in the sense that it is not caused by the limitations of the measuring instrument; the observed power-law PDS is an intrinsic property of the GRB itself. Nonetheless, we can remove this contribution much as we would eliminate red $(\alpha=2)$ or pink $(0<\alpha<2)$ noise caused by our measuring device (e.g., Vaughan 2005).

To estimate the power-law slope, we construct a new PDS for GRB 090709A, including all of the available 15-350 keV BAT data and without any detrending. This helps to remove the noise at $P \gtrsim 10 \mathrm{~s}$ and provides a longer lever arm to calculate the PDS slope. For GRB 090709A, we then find $\alpha \approx 2.5$.

In the right panel of Figure 2, we renormalize the PDS of GRB 090709A using the underlying spectrum described above (solid line). While the same shape is visible in the PDS, the significance of the peak has dropped dramatically. After correcting for the number of trials, we find that the feature at $P=8.1 \mathrm{~s}$ is significant at only the $\sim 2 \sigma$ level.

As stated previously, the inferred significance depends sensitively on the assumed noise properties. The value we derive for GRB 090709A ( $\alpha=2.5)$ is significantly steeper than the average BATSE value of $\sim 5 / 3$ (Beloborodov et al. 1998). To investigate further, we have performed a comparable analysis on all BAT GRBs with a fluence at least $70 \%$ of the value derived for GRB 090709A (to provide sufficient S/N) and a duration $t_{90}>70 \mathrm{~s}$ (to provide sufficient sensitivity to $P \approx 10 \mathrm{~s}$ ). Only six additional events meet these criteria: GRBs 041223, 061007, 080319B, 080607, 090201, and 090618. The results of this analysis are shown in Table 1 and Figure 3.

Each event in this sample exhibits a peak in the PDS with period $\sim 10 \mathrm{~s}$ and significance greater than $2 \sigma$ when normalizing by a flat PDS. However, the significance of these features drops dramatically when normalizing by a power-law spectrum with $\alpha \approx 2-2.5$.
Table 1

Power Density Spectra of Bright Swift-BAT GRBs

\begin{tabular}{lcccc}
\hline \hline GRB & $\begin{array}{c}\text { Period }^{\mathrm{a}} \\
(\mathrm{s})\end{array}$ & $\begin{array}{c}\text { White } \mathrm{S} / \mathrm{N}^{\mathrm{b}} \\
(\sigma)\end{array}$ & PDS Slope & $\begin{array}{c}\text { Red S/N } \\
(\sigma)\end{array}$ \\
\hline $090709 \mathrm{~A}$ & 8.1 & 14.1 & -2.5 & 5.0 \\
041223 & 16.5 & 2.0 & -2.6 & 0.4 \\
061007 & 7.6 & 4.8 & -2.2 & 1.2 \\
$080319 \mathrm{~B}$ & 8.3 & 4.6 & -2.3 & 0.8 \\
080607 & 7.7 & 7.3 & -2.2 & 2.7 \\
090201 & 8.3 & 8.8 & -1.9 & 3.5 \\
090618 & 15.2 & 3.1 & -2.2 & 1.2 \\
\hline
\end{tabular}

Notes.

${ }^{a}$ Period of the strongest feature observed in the PDS.

${ }^{\mathrm{b}}$ Single-trial S/N of the strongest feature observed in the PDS assuming a flat (white) intrinsic spectrum across the entire frequency range (normalized to the region $P=3-6.5 \mathrm{~s}$ ).

${ }^{c}$ Single-trial $\mathrm{S} / \mathrm{N}$ of the strongest feature observed in the PDS assuming an intrinsic power-law spectrum (red noise). See the text for details.

If we instead assume the slope of the PDS of GRB 090709A is equal to the median value derived for BATSE GRBs (i.e., $\alpha=5 / 3$ ), we find that the significance of the feature at $P=8.1 \mathrm{~s}$ increases somewhat to $\lesssim 3 \sigma$. We stress that this scenario provides a relatively conservative estimate for the QPO significance: though the PDS slope of GRBs can vary over a relatively large range $(0.5 \lesssim \alpha \lesssim 3.0$; Suzuki et al. 2002), Beloborodov et al. (2000) have demonstrated a steepening of the observed power law at lower energies. Given the somewhat softer bandpass of the Swift-BAT, it is not entirely surprising, then, that the PDS slopes derived for Swift events fall on average on the steeper end of the BATSE distribution. Yet even if we assume $\alpha=5 / 3$, the peak observed in GRB 090709A (as well as all the other Swift events examined here) is not particularly significant.

The apparent periodicity of GRB 090709A was also reported by several additional gamma-ray satellites (Golenetskii et al. 2009; Gotz et al. 2009; Ohno et al. 2009), presumably performing a similar analysis to that of Markwardt et al. (2009). 


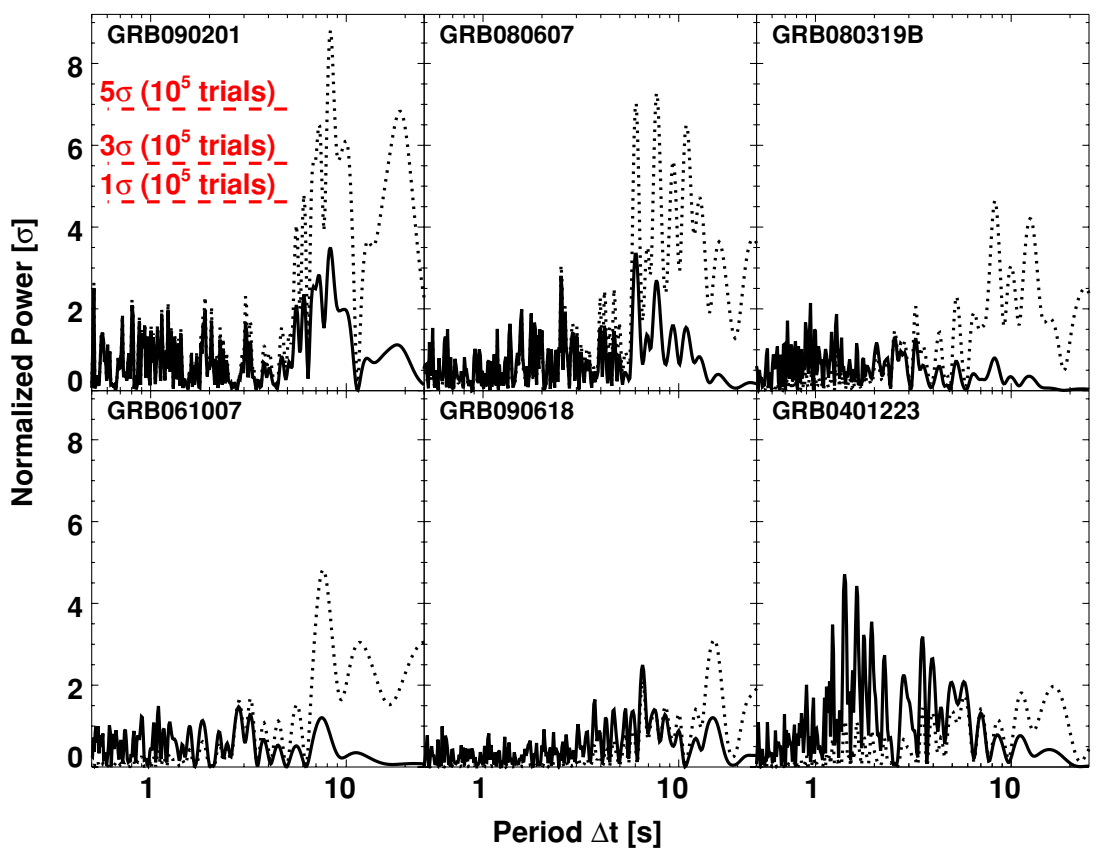

Figure 3. PDSs of other Swift long-duration GRBs. We have selected all BAT events with fluence $>70 \%$ that of GRB 090709A and duration $t_{90}>70 \mathrm{~s}$. As in the right panel of Figure 2, the dashed lines plot the normalized PDS assuming a flat underlying spectrum using the noise properties in the range $3<P<6.5 \mathrm{~s}$. All six events have a peak at $P \approx 10 \mathrm{~s}$ with single-trial significance $>2 \sigma$, though none is as strong as that derived for GRB 090709A. After correcting for the underlying power-law spectrum (solid lines) and the number of trials, none of these features remain with significance $>3 \sigma$.

(A color version of this figure is available in the online journal.)

We repeated our analysis on the publicly available $S u z a k u$ light curve of GRB 090709A and find our results essentially unchanged: after normalizing by a power law with $\alpha \approx 2.5$, the significance of the feature at $P=8.1 \mathrm{~s}$ falls to $\sim 2-3 \sigma$.

De Luca et al. (2010) have performed a similar timing analysis of both the Swift-BAT and Integral-API/ACS (Gotz et al. 2009) light curves of GRB 090709A. These authors confirm our primary result that the reported periodicity in the BAT light curve at $P=8.1 \mathrm{~s}$ is only significant at the $\lesssim 3 \sigma$ level. Interestingly, the signal at this frequency appears to be much weaker in the SPI-ACS data.

To summarize, while the high-energy light curve of GRB 090709A shows tantalizing evidence of QPOs with $P=$ $8.1 \mathrm{~s}$, we do not take it to be a significant feature required by the data. It is certainly the most compelling candidate for such behavior among the long-duration GRB population observed by Swift. However, when properly accounting for the shape of the underlying spectrum, we find that the significance of the observed periodicity is not sufficiently large to be conclusive. Instead, we must look to the afterglow and environment of GRB 090709A to attempt to unveil its progenitor.

\section{FOLLOW-UP OBSERVATIONS}

The XRT (Burrows et al. 2005) on board Swift began observing the field of GRB 090709A beginning at $t_{0}+74 \mathrm{~s}$. A fading X-ray counterpart inside the BAT error circle was promptly identified and reported to the community (Morris et al. 2009; Osborne et al. 2009; Rowlinson \& Morris 2009). Crossmatching field sources detected by the XRT with cataloged nearinfrared (NIR) positions from the Two Micron All Sky Survey (2MASS; Skrutskie et al. 2006), we measure a localization for the afterglow of $\alpha=19^{\mathrm{h}} 19^{\mathrm{m}} 42^{\prime \prime} .46, \delta=+60^{\circ} 43^{\prime} 39^{\prime \prime} .6$, with a $90 \%$ containment radius of 1".2 (J2000.0; see Butler 2007 for details).
We plot the X-ray afterglow of GRB 090709A in Figure 6, which we have taken from the online compilation of N.R.B. ${ }^{11}$ Like many GRBs in the Swift era, the XRT began observations of GRB 090709A while the prompt gamma-ray emission was still ongoing, and the earliest X-ray observations extrapolate smoothly to the tail of the prompt emission. After $10^{3} \mathrm{~s}$, the $\mathrm{X}$-ray light curve is well described by a single power-law decay with index $\alpha_{X}=1.38 \pm 0.02\left(\chi^{2}=394.93\right.$ for 417 dof $)$.

Our derived temporal slope falls in between the two values reported by De Luca et al. (2010), who adopt a broken powerlaw fit with $\alpha_{X, 1}=1.15 \pm 0.01, \alpha_{X, 2}=1.48 \pm 0.05$, and $t_{\mathrm{b}} \approx 0.26$ day. Because we do not include the initial X-ray observations at $t<10^{3} \mathrm{~s}$ (when the decay was shallower) in our fit, we do not require a break in the X-ray light curve. However, there is a large gap in the X-ray coverage from $t \approx 2$ days to $t \approx 10$ days. A broken power law with $2 \lesssim t_{b} \lesssim 10$ days can also provide a reasonable fit. In this case, the initial decay index is somewhat more shallow $\left(\alpha_{1, X} \approx 1.2\right)$; the break time and post-break decay index are not well constrained.

The X-ray spectrum at $t>10^{4} \mathrm{~s}$ is adequately fit by a power law with index $\beta_{X}=0.95 \pm 0.07\left(\chi^{2}=178.58\right.$ for $175 \mathrm{dof})$, although the inferred X-ray column $\left(N_{\mathrm{H}}=\right.$ $1.83_{-0.21}^{+0.24} \times 10^{21} \mathrm{~cm}^{-2}$ at $\left.z=0\right)$ is significantly in excess of the Galactic value $\left(N_{\mathrm{H}, \mathrm{Gal}}=6.6 \times 10^{20} \mathrm{~cm}^{-2}\right.$; Kalberla et al. 2005). In the case of an intrinsic power-law spectrum, the requirement for extinction in excess of the Galactic value is significant at the $13.5 \sigma$ level. Fits with only Galactic extinction require at least two blackbody components and still provide worse quality than an absorbed power law $\left(k T_{1}=0.32 \pm 0.02 \mathrm{keV}\right.$, $k T_{2}=1.11 \pm 0.05 \mathrm{keV} ; \chi^{2}=198.64$ for $\left.174 \mathrm{dof}\right)$. These results are broadly consistent with those reported by Rowlinson $\&$ Morris (2009) and De Luca et al. (2010).

\footnotetext{
11 http://astro.berkeley.edu/ nat/swift; see Butler \& Kocevski (2007) for details.
} 
Table 2

Optical/NIR Observations of GRB 090709A

\begin{tabular}{|c|c|c|c|c|c|c|}
\hline $\begin{array}{c}\text { Observation Date } \\
(2009 \text { UT }) \\
\end{array}$ & $\begin{array}{c}\text { Time Since Burst }{ }^{\mathrm{a}} \\
\text { (s) }\end{array}$ & Telescope & Filter & $\begin{array}{c}\text { Exposure Time } \\
\text { (s) }\end{array}$ & Magnitude $^{b}$ & Reference \\
\hline Jul 9 07:40:22 & 120.0 & PAIRITEL & $H$ & 23.4 & $15.52 \pm 0.16$ & $*$ \\
\hline Jul 9 07:40:22 & 120.0 & PAIRITEL & $K_{s}$ & 23.4 & $14.48 \pm 0.21$ & $*$ \\
\hline Jul 9 07:40:26 & 142.5 & P60 & $r^{\prime}$ & 60.0 & $21.80 \pm 0.51$ & $*$ \\
\hline Jul 9 07:40:58 & 177.0 & PAIRITEL & $H$ & 46.8 & $>15.90$ & $*$ \\
\hline Jul 9 07:40:58 & 177.0 & PAIRITEL & $K_{s}$ & 46.8 & $14.25 \pm 0.14$ & $*$ \\
\hline Jul 9 07:42:17 & 307.5 & PAIRITEL & $H$ & 117.0 & $16.20 \pm 0.13$ & $*$ \\
\hline Jul 9 07:42:17 & 307.5 & PAIRITEL & $K_{s}$ & 117.0 & $15.00 \pm 0.15$ & $*$ \\
\hline Jul 9 07:43:17 & 313.2 & P60 & $z^{\prime}$ & 60.0 & $19.54 \pm 0.30$ & $*$ \\
\hline Jul 9 07:40:58 & 383.5 & PAIRITEL & $J$ & 304.2 & $18.43 \pm 0.28$ & $*$ \\
\hline Jul 9 07:43:53 & 398.7 & P60 & $r^{\prime}$ & 180.0 & $22.24 \pm 0.42$ & $*$ \\
\hline Jul 9 07:45:08 & 484.0 & P60 & $i^{\prime}$ & 180.0 & $>21.62$ & $*$ \\
\hline Jul 9 07:46:33 & 569.4 & P60 & $z^{\prime}$ & 180.0 & $>19.92$ & $*$ \\
\hline Jul 9 07:45:20 & 680.0 & PAIRITEL & $H$ & 351.0 & $16.88 \pm 0.16$ & $*$ \\
\hline Jul 9 07:45:20 & 680.0 & PAIRITEL & $K_{s}$ & 351.0 & $15.89 \pm 0.21$ & $*$ \\
\hline Jul 9 07:46:07 & $1.637 \times 10^{3}$ & FTN & $i^{\prime}$ & 520.0 & $>20.6$ & 1 \\
\hline Jul 9 07:41:16 & $1.737 \times 10^{3}$ & FTN & $R$ & 720.0 & $22.7 \pm 0.5$ & 1 \\
\hline Jul 9 08:06:34 & $2.040 \times 10^{3}$ & P60 & $r^{\prime}$ & 720.0 & $>22.81$ & $*$ \\
\hline Jul 9 08:11:06 & $2.251 \times 10^{3}$ & P60 & $i^{\prime}$ & 600.0 & $>22.36$ & $*$ \\
\hline Jul 9 08:13:11 & $2.377 \times 10^{3}$ & P60 & $z^{\prime}$ & 600.0 & $>20.75$ & $*$ \\
\hline Jul 9 08:20:17 & $2.743 \times 10^{3}$ & P60 & $g^{\prime}$ & 480.0 & $>22.09$ & $*$ \\
\hline Jul 9 09:30:00 & $6.686 \times 10^{3}$ & Subaru/MOIRCS & $K$ & $\ldots$ & 18.2 & 2 \\
\hline Jul 9 22:37:55 & $5.479 \times 10^{4}$ & NOT/ALFOSC & $R$ & 1800.0 & $>24.0$ & 3 \\
\hline Jul 9 23:46:42 & $6.488 \times 10^{4}$ & $\mathrm{CAHA} \mathrm{OMEGA}_{2000}$ & $H$ & 3600.0 & $>20.73$ & $*$ \\
\hline Jul 10 00:48:09 & $6.777 \times 10^{4}$ & CAHA/OMEGA 2000 & $K_{s}$ & 4140.0 & $20.77 \pm 0.39$ & $*$ \\
\hline Jul 10 01:57:43 & $6.801 \times 10^{4}$ & CAHA/OMEGA 2000 & $J$ & 3120.0 & $>21.74$ & $*$ \\
\hline Jul 11 00:45:00 & $1.480 \times 10^{5}$ & GTC/OSIRIS & $i^{\prime}$ & 2100.0 & $>25.5$ & $*$ \\
\hline Jul 17 10:06:16 & $7.009 \times 10^{5}$ & Keck II/NIRC2 & $K^{\prime}$ & 1500.0 & $>22.81$ & $*$ \\
\hline
\end{tabular}

Notes.

a Time elapsed from the Swift-BAT trigger, 07:38:34 UT on 2009 July 9 to the midpoint of the exposure.

b Magnitudes are provided in the AB system (Oke \& Gunn 1983) for the $g^{\prime} r^{\prime} i^{\prime} z^{\prime}$ filters and in the Vega system for the remaining filters. Errors quoted are $1 \sigma$ photometric and instrumental errors summed in quadrature. For measurements with significance $<2 \sigma, 3 \sigma$ upper limits are provided. Galactic extinction $(E(B-V)=0.09$ mag; Schlegel et al. 1998) has not been incorporated in the reported magnitudes.

References. (*) This work; (1) Guidorzi et al. 2009; (2) Aoki et al. 2009; (3) Malesani et al. 2009.

Both the Peters Automated Infrared Telescope (PAIRITEL; Bloom et al. 2006a) and the robotic Palomar 60 inch $(1.5 \mathrm{~m})$ telescope (P60; Cenko et al. 2006) automatically responded to the Swift alert and began observations within 2 minutes of the trigger time. Additional observations were obtained at later times with the OMEGA 2000 NIR camera on the $3.5 \mathrm{~m}$ telescope at Calar Alto Observatory (2009 July 10), with the OSIRIS instrument (J. Cepa et al. 2010, in preparation) attached to the $10.4 \mathrm{~m}$ Gran Telescopio Canarias (GTC) at the IAC's Observatorio del Roque de los Muchachos in La Palma (2009 July 11), and with NIRC2 behind the laser guide star adaptive optics system (Wizinowich et al. 2006) on the $10 \mathrm{~m}$ Keck II telescope (2009 July 17). All images were processed using standard routines in the IRAF ${ }^{12}$ environment. Photometric calibration was performed relative to the USNO-B1.0 catalog (Monet et al. 2003) in the optical, using the filter transformations of Jordi et al. (2006) where appropriate, and relative to 2MASS (Skrutskie et al. 2006) in the NIR. The results of this campaign are listed in Table 2 and displayed in Figure 6.

Morgan et al. (2009) reported the PAIRITEL afterglow detection in the $H$ and $K_{s}$ filters; subsequent reanalysis has revealed a detection in the $J$ band as well (Figure 4). Using

\footnotetext{
12 IRAF is distributed by the National Optical Astronomy Observatory, which is operated by the Association for Research in Astronomy, Inc., under cooperative agreement with the National Science Foundation.
}

early-time $K_{s}$-band images and reference objects from the 2MASS catalog, we measure a position for the afterglow of $\alpha=19^{\mathrm{h}} 19^{\mathrm{m}} 42^{\prime \prime} .64, \delta=+60^{\circ} 43^{\prime} 39^{\prime \prime}$, , with a $90 \%$ containment radius of $0{ }^{\prime \prime} 4(\mathrm{~J} 2000.0)$. This location is at a distance of 1 .". 4 from the center of the X-ray error circle, consistent within the $90 \%$ confidence regions.

Combining the PAIRITEL NIR detections with the marginal $r^{\prime}$ and $z^{\prime}$ detections from P60, we find that the afterglow was extremely red. After correcting for Galactic extinction $(E[B-V]=0.09 \mathrm{mag}$; Schlegel et al. 1998), we fit the nearly simultaneous $r^{\prime} z^{\prime} J H K_{s}$ photometry at $t \approx 350 \mathrm{~s}$ to a power-law spectral energy distribution (SED) model and find an extremely steep spectral index: $\beta_{O}=3.8 \pm 0.3\left(\chi^{2}=4.16\right.$ for 3 dof $)$. The temporal decay index in the optical is not very well constrained by our observations; assuming the same value for all filters, we find $\alpha_{O} \approx 0.9$.

We observed the field of GRB 090709A with the Very Large Array (VLA) $)^{13}$ at a frequency of $8.46 \mathrm{GHz}$ on July 11.42 and at $1.43 \mathrm{GHz}$ on July 11.39. For both observations the array was in the "C" configuration. The flux-density scale was tied to 3C 286 or 3C 147 and the phase was measured by switching between the GRB and a nearby, bright, point-source calibrator.

\footnotetext{
13 The VLA is operated by the National Radio Astronomy Observatory, a facility of the National Science Foundation operated under cooperative agreement by Associated Universities, Inc.
} 

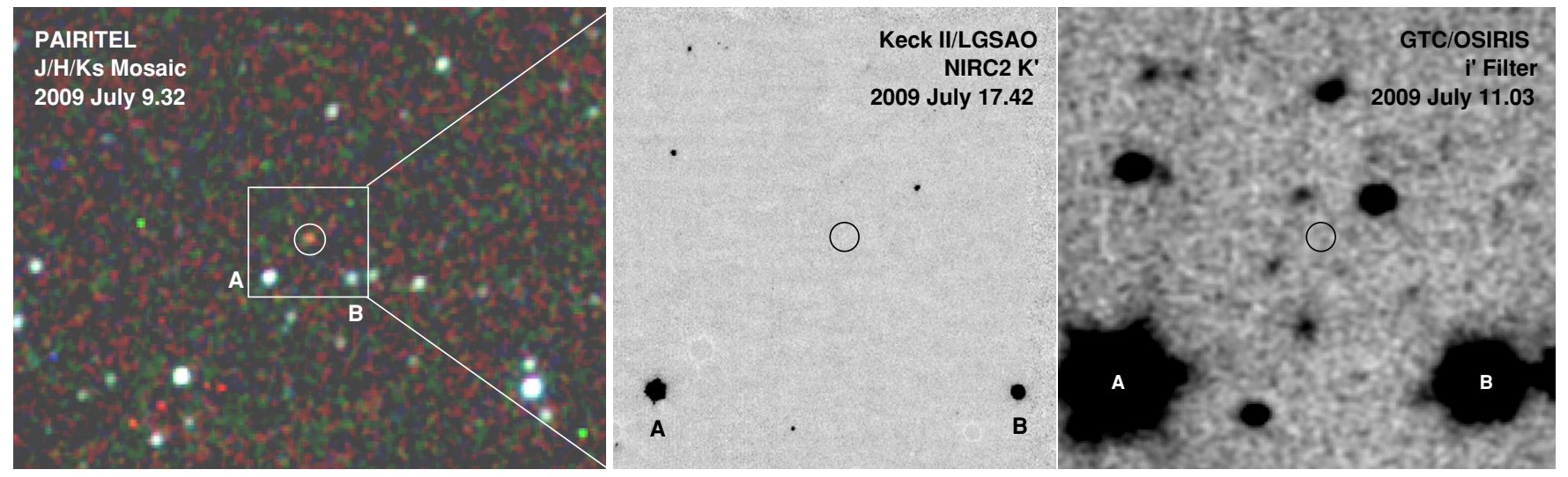

Figure 4. Left: PAIRITEL false-color $\left(J H K_{s}\right)$ image of the field of GRB 090709A, taken at a mean epoch of $t_{0}+379 \mathrm{~s}$. The afterglow is centered inside the white circle ( $5^{\prime \prime}$ radius) and is clearly much redder than the surrounding field stars. Stars $A$ and $B$ are marked for reference in all images. Center: Keck/NIRC2 $K^{\prime}$ image of the same field, approximately 1 week later. The black circle is the $90 \%$ confidence XRT localization (1". 2 radius). Right: GTC/OSIRIS $i^{\prime}$ image of the identical field on 2009 July 11 (smoothed with a 4 pixel Gaussian filter). The XRT error circle is again shown in black. No sources are visible in the immediate environment ( $\left.\lesssim 4^{\prime \prime}\right)$ of the afterglow location to a $3 \sigma$ limiting magnitude of $K^{\prime}>22.8 \mathrm{mag}, i^{\prime}>25.5 \mathrm{mag}$. All images are oriented with north up and east to the left.

(A color version of this figure is available in the online journal.)

To maximize sensitivity, the full VLA continuum bandwidth $(100 \mathrm{MHz})$ was recorded in two $50 \mathrm{MHz}$ bands. Data reduction was carried out following standard practice in the AIPS software package. No emission is detected at the NIR afterglow location at either frequency to $2 \sigma$ limits of $f_{\nu}(8.46 \mathrm{GHz})<70 \mu \mathrm{Jy}$, $f_{v}(1.43 \mathrm{GHz})<288 \mu \mathrm{Jy}$ (see also Chandra \& Frail 2009).

Finally, we have obtained a series of pre-GRB optical images with the Palomar 48 inch $(1.2 \mathrm{~m})$ telescope from the Deep Sky catalog. ${ }^{14}$ Thirty-two images of the field of GRB 090709A were taken as part of the Palomar-QUEST survey (Djorgovski et al. 2008) over a time interval of a month in 2005 August. The images were obtained using an order-blocking filter with a cutoff blueward of $\lambda \approx 6100 \AA$, which we calibrate relative to the Sloan Digital Sky Survey $i^{\prime}$ band. A coadded image of these frames does not reveal any source at the location of the afterglow of GRB 090709A to a $3 \sigma$ limit of $i^{\prime}>23.2 \mathrm{mag}$.

\section{A NEW SOFT GAMMA-RAY REPEATER?}

The detection of QPOs with $P=8.1 \mathrm{~s}$ would strongly suggest an SGR origin. Unlike normal (rotation-powered) pulsars, the spin periods of known SGRs and AXPs fall in the range $2.0-11.8 \mathrm{~s}^{15}{ }^{15}$ We therefore consider if the observed properties (outside the prompt high-energy emission) could themselves be consistent with an SGR flare.

By definition SGRs undergo repeated high-energy outbursts. We have therefore searched the historical GRB catalogs of both the Interplanetary Network (IPN; Laros et al. 1997; Hurley et al. 1999a) and BATSE for an excess of localizations consistent with the position of GRB 090709A (both missions provided relatively coarse angular localizations, requiring such a statistical analysis; see Ofek 2007 for details). Using the IPN catalog from 1990 November 12 to 2005 October $31,{ }^{16}$ we found four IPN localizations consistent with the position of GRB 090709A. However, running the same search on random pointings with the same ecliptic latitude results in a median overlap of four GRBs.

\footnotetext{
14 See http://supernova.lbl.gov/ nugent/deepsky.html.

15 See, for example, the McGill SGR/AXP Online Catalog at http://www.physics.mcgill.ca/ pulsar/magnetar/main.html.

16 See http://www.ssl.berkeley.edu/ipn3/interpla.html; version 2005

December 17. For reference, this IPN catalog version is also available at

http://astro.caltech.edu/ eran/GRB/IPN/NearbyGal/CatIPN.txt.ver17122005.
}

A similar analysis with short-duration $\left(t_{90}<1 \mathrm{~s}\right)$ BATSE GRBs in the current catalog ${ }^{17}$ also reveals no significant excess in the direction of GRB 090709A.

SGR flares are broadly divided into three classes (e.g., Woods $\&$ Thompson 2006; Mereghetti 2008). The rarest of these are the giant flares, with only a handful observed to date (e.g., Mazets et al. 1979; Hurley et al. 1999b; Feroci et al. 1999; Mazets et al. 1999; Hurley et al. 2005). The high-energy light curves of giant flares are dominated by a short ( $\sim 1 \mathrm{~s})$, hard spike of gamma rays. Thereafter, the emission softens and decays exponentially with QPOs at the spin period of the underlying NS. Clearly the bright initial spike was not observed from GRB 090709A. However, it is possible that this component could be anisotropic and beamed away from our line of sight, as some bright SGR flares lacking the initial spike have been observed (e.g., Mereghetti et al. 2009).

Giant SGR flares have gamma-ray energy releases $\sim 10^{44}$ $10^{46} \mathrm{erg}$. Using our derived $15-350 \mathrm{keV}$ fluence (Section 2), the observed system would fall at a distance of $\sim 0.1-1 \mathrm{Mpc}$ (note that any beaming correction would only make the event more nearby, a possibility we discuss subsequently). Such distances imply an origin either in the halo of the Milky Way or in a nearby galaxy in the Local Group.

Magnetars have relatively short characteristic ages $(P / \dot{P} \approx$ $10^{3}-10^{5} \mathrm{yr}$ ), which would seem to rule out a halo origin if the NS formed directly from the core collapse of a massive star. If the progenitor system were associated with an older stellar population, for instance the accretion-induced collapse of a white dwarf (Canal \& Schatzman 1976), this could perhaps explain the large offset from a site of recent star formation. However, a relatively unphysical model would be required to account for the observed X-ray spectrum without excess extinction (see below).

Giant magnetar flares from nearby galaxies are now believed to account for some fraction of the observed BATSE short bursts (e.g., Hurley et al. 2005; Gaensler et al. 2005; Cameron et al. 2005; Tanvir et al. 2005; Ofek et al. 2006, 2008; Ofek 2007; Mazets et al. 2008; Hurley et al. 2010; Chapman et al. 2009). In the case of GRB 090709A, however, the lack of an obvious host-galaxy candidate seems to disfavor an extragalactic (but nearby; $d \lesssim 1 \mathrm{Mpc}$ ) origin. Our pre- (Deep Sky) and

\footnotetext{
${ }^{17}$ See http://www.batse.msfc.nasa.gov/batse/grb/catalog/current.
} 
post- (NIRC2) GRB limits at the location of GRB 090709A correspond to absolute magnitudes of $M_{i^{\prime}}(\mathrm{AB}) \gtrsim-2 \mathrm{mag}$, $M_{K^{\prime}}$ (Vega) $\gtrsim-2 \mathrm{mag}$ for $d \approx 1 \mathrm{Mpc}$, sufficient to detect individual supergiant stars, let alone dwarf galaxies (modulo extinction). The post-GRB $i^{\prime}$ limits from the GTC provide even tighter constraints.

Even with a large natal kick velocity $\left(10^{4} \mathrm{~km} \mathrm{~s}^{-1}\right)$, the short lifetime limits the distance a magnetar can travel away from the host-galaxy disk (where it was presumably formed) to $\lesssim 1 \mathrm{kpc}$. For $d \approx 1 \mathrm{Mpc}$, this corresponds to an angular offset of $\sim 3^{\prime}$. No Local Group galaxy is known within $10^{\circ}$ of GRB 090709A. The lack of any observed candidate host galaxy at this location seems to independently rule out a giant magnetar flare having extragalactic origin.

Intermediate magnetar flares have durations of order $1 \mathrm{~s}$ or longer and energy releases $\sim 10^{41}-10^{43} \mathrm{erg}$. With the observed fluence of GRB 090709A, this corresponds to distances of $\sim 4-40 \mathrm{kpc}$. The primary drawback with this scenario is the location of GRB 090709A with respect to the Galactic plane. With Galactic coordinates $(l, b)=(91.8,20.1)$, a total distance of $\gtrsim 4 \mathrm{kpc}$ corresponds to a height of $\gtrsim 1 \mathrm{kpc}$ above the Galactic plane. Of the 18 known and suspected magnetars, only two have Galactic latitudes $|b|>2^{\circ}$ : SGR 0525-66 (in the Large Magellanic Cloud (LMC); Mazets et al. 1982; Kulkarni et al. 2003; Klose et al. 2004), and CXOU J010043.1-721134 (in the Small Magellanic Cloud (SMC); Lamb et al. 2002). ${ }^{18}$ For those SGRs in our Galaxy with known distances, the typical scale height is $\sim 100 \mathrm{pc}$, as would be expected from their young ages. Furthermore, several are associated with supernova remnants (Klose et al. 2004; Gelfand \& Gaensler 2007; Gaensler et al. 1999; Fahlman \& Gregory 1981; Vasisht \& Gotthelf 1997) or young stellar clusters (Vrba et al. 2000; Corbel \& Eikenberry 2004; Muno et al. 2006), so even in the absence of a planar birth, some remnant of recent star formation should be evident nearby.

Finally, we consider SGR flares with energy release $<10^{41} \mathrm{erg}$. Such flares are quite common, though typically observed with durations $\sim 100 \mathrm{~ms}$. The spectra of these flares are well described by an optically thin thermal bremsstrahlung model, with $k T \approx 20-40 \mathrm{keV}$, unlike the observed properties of GRB 090709A. If we require a scale height of $\lesssim 100 \mathrm{pc}$, this limits the total distance to GRB 090709A to be $\lesssim 300$ pc. The energy release would therefore be $\lesssim 5 \times 10^{38} \mathrm{erg}$, consistent with observations of low-level flaring activity from known SGRs and AXPs. However, the small distance would present additional difficulties. Nearly all sufficiently well-localized magnetars exhibit quiescent X-ray emission at a luminosity of $10^{33}-10^{36} \mathrm{erg} \mathrm{s}^{-1}$. At $d \lesssim 300 \mathrm{pc}$, such emission should have been detected by $\mathrm{X}$-ray surveys such as the ROSAT All-Sky Survey (Voges et al. 1999), yet no cataloged X-ray sources are consistent with this position.

Finally, we note that all Galactic models, even if associated with an older population and not subject to the requirement of nearby star formation, would struggle to explain the excess extinction inferred from the X-ray spectrum and optical SED. While the intrinsic X-ray and optical spectra need not be power laws, we find no physically motivated models capable of reproducing the observed X-ray spectrum and optical SED absent an excess absorption component.

\footnotetext{
18 The localization of an additional SGR candidate, SGR $0418+5729$, is centered at $b=5$. 1 , but the error circle has a radius of $\sim 7^{\circ}$ (van der Horst et al. 2009).
}

\section{MASSIVE STAR CORE COLLAPSE}

Setting aside again the high-energy light curve, we consider the long-lived afterglow emission associated with GRB 090709A. Radio afterglow emission has been observed from two giant SGR flares (Frail et al. 1999; Cameron et al. 2005; Gaensler et al. 2005), and fading X-ray counterparts are relatively common, even for intermediate flares (e.g., Mereghetti 2008). However, the physical mechanism underlying this emission is thought to be quite different from the process powering long-duration GRB afterglows.

In the case of magnetar flares, afterglow emission results from a rapid injection of relativistic particles into the circumburst medium. The particles expand adiabatically, and, consequently, the light curves decline quite rapidly with time (power-law indices $\alpha \gtrsim 2.5$; Frail et al. 1999; Cheng \& Wang 2003; Cameron et al. 2005; Gaensler et al. 2005). For GRB afterglows, a relativistic shock wave accelerates electrons in the circumburst medium, causing them to emit synchrotron radiation (e.g., Piran 2005). As the shock expands outward, it continues to excite electrons further and further away from the explosion site, resulting in additional emission from larger radii and a slower fading rate $(\alpha \approx 1-2$; Sari et al. 1998).

A detailed comparison of well-sampled, broadband light curves may therefore be able to distinguish between these two scenarios. In the case of GRB 090709A, however, the lack of optical/NIR and radio data makes such a comparison difficult. We therefore attempt to answer a simpler question: is the observed emission consistent with standard GRB afterglow models?

To do so, we require some knowledge of the distance to GRB 090709A. Grupe et al. (2007) have demonstrated that the detection of X-ray absorption in excess of the Galactic value can be used to place a probabilistic upper limit on the distance to GRBs; the steep redshift dependence of soft X-ray absorption $\left(\sim(1+z)^{8 / 3}\right)$ effectively precludes the observation of distant events with large column densities. Using Equation (1) from Grupe et al. (2007) and our derived X-ray column excess, we find $z \lesssim 3.5$. We caution, however, that some well-observed events are known to violate this redshift limit (e.g., GRB 080607; Prochaska et al. 2009).

A more strict limit can be placed using the P60 and Faulkes Telescope (Guidorzi et al. 2009) $r^{\prime}$-band afterglow detections. If we assume that the Ly $\alpha$ break in the GRB rest frame falls at the red edge of the observed $R$-band filter, we conclude $z \lesssim 4.5$.

An alternate approach is to use the observed BAT and XRT luminosity functions as a guide. The left panel of Figure 5 displays the X-ray afterglow flux $(0.3-10 \mathrm{keV})$ at a common time of $t=1$ day (observer frame) for all Swift GRBs to date with known redshifts. The X-ray afterglow of GRB 090709A is one of the brightest in the Swift era, well above any previous event with $z>4$.

Much like the X-ray flux, the BAT fluence from GRB 090709A is also one of the largest ever seen by Swift. In the right panel of Figure 5, we plot the rest-frame $1-10^{4} \mathrm{keV}$ isotropic energy release $\left(E_{\gamma, \text { iso }}\right)$ for all BAT GRBs with known redshifts, along with $K$-corrected values for GRB 090709A at $z=1,2,3,4$, and 5. To be consistent with the observed BAT distribution, GRB 090709A should fall at $z \lesssim 3$. More quantitatively, applying the GRB world model from Butler et al. (2010), we find $z<2.5(z<1)$ at $99 \%(90 \%)$ confidence.

An approximate lower limit can be derived using the lack of a host candidate at the location of the afterglow of GRB 090709A. 

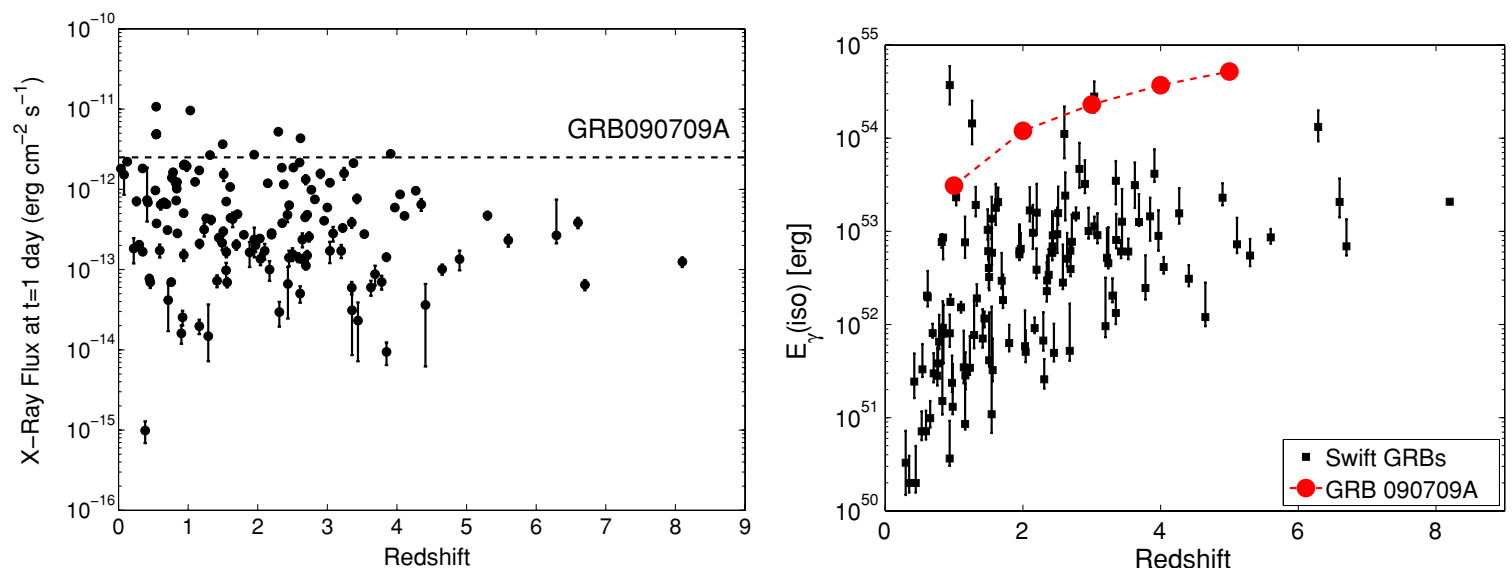

Figure 5. Left: $0.3-10 \mathrm{keV}$ flux at a common time of $t=1$ day (observer frame) for all Swift GRBs with known redshift. GRB 090709A is indicated with a dashed horizontal line. Right: prompt gamma-ray $\left(1-10^{4} \mathrm{keV}\right.$ rest frame) energy release for all Swift GRBs with known redshift. GRB 090709A is shown for $z=1,2,3,4$, and 5. If, as suggested by De Luca et al. (2010), GRB 090709A lies at $z \approx 4$, it would be one of the brightest events ever detected by the Swift satellite.

(A color version of this figure is available in the online journal.)

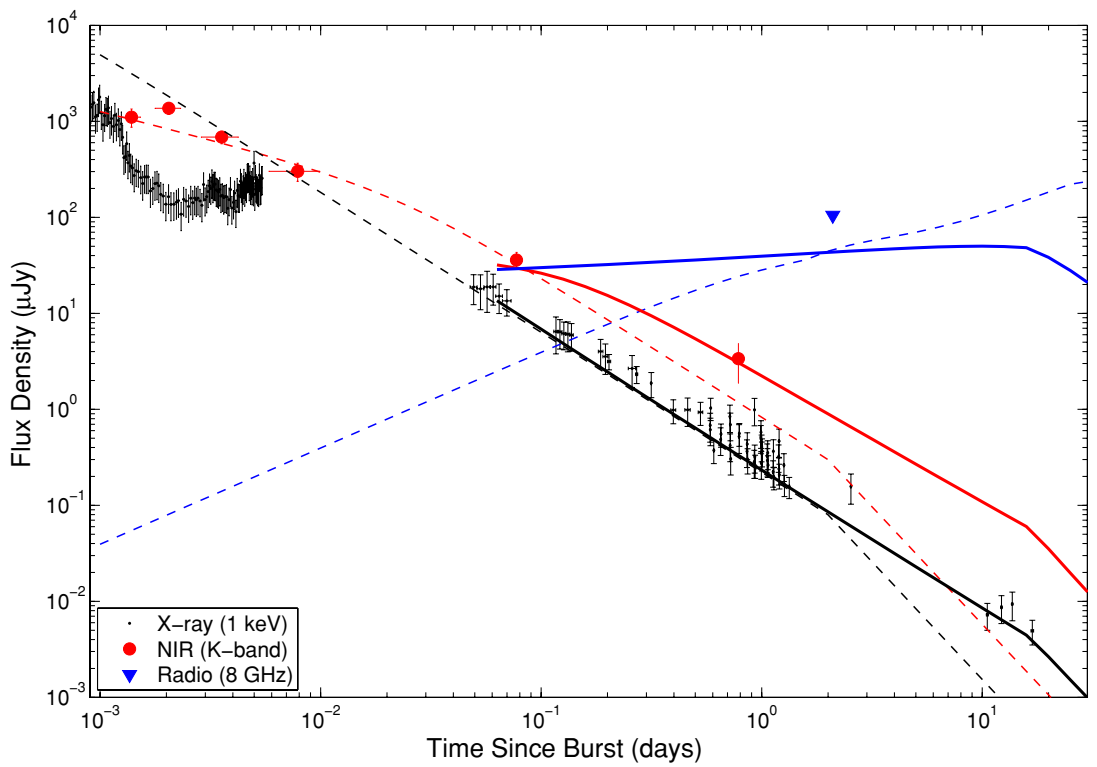

Figure 6. X-ray, $K$-band, and radio afterglow of GRB 090709A. The dashed lines indicate a model that attempts to explain the entirety of the observations (the model has the following parameters: $E_{\mathrm{KE} \text {,iso }}=1.5 \times 10^{53} \mathrm{erg}, \theta=0.2, p=2.01, \epsilon_{e}=0.5, \epsilon_{B}=0.2, z=1, n=40 \mathrm{~cm}^{-3}, A_{K, \mathrm{obs}}=6$ mag). While this model can account for most of the observed features, it implies an extremely small cooling frequency $\left(v_{c}<v_{O}\right.$ for $t<0.01$ day). The solid lines show a model with more reasonable physical parameters designed to explain the late-time $\left(t \gtrsim 0.1\right.$ day) data (the model has the following parameters: $E_{\mathrm{KE}, \text { iso }}=1.5 \times 10^{53} \mathrm{erg}, \theta=0.1, p=2.06$, $\epsilon_{e}=0.6, \epsilon_{B}=0.1, z=1, n=0.01 \mathrm{~cm}^{-3}, A_{K, \text { obs }}=3 \mathrm{mag}$ ). The lack of optical and radio coverage at these times makes such models highly degenerate.

(A color version of this figure is available in the online journal.)

With only a handful of exceptions (e.g., Cenko et al. 2008), the observed host-offset distribution of long-duration GRBs is limited to $r \lesssim 10 \mathrm{kpc}$ (Bloom et al. 2002; Fruchter et al. 2006). Long-duration GRB hosts are typically blue, irregular galaxies (e.g., Fruchter et al. 2006; Wainwright et al. 2007) with low metallicity (e.g., Stanek et al. 2006) and large specific star-formation rates (e.g., Christensen et al. 2004). If we take the low-metallicity dwarf SMC as a proxy for our GRB host $\left(M_{V} \approx-16.6 \mathrm{mag}\right)$, the nondetection in the $i^{\prime}$ band with the GTC requires $z \gtrsim 0.5$.

De Luca et al. (2010) attempt to constrain the distance to GRB 090709A via the Swift-XRT and XMM-Newton-EPIC $\mathrm{X}$-ray afterglow spectra. Assuming an intrinsic power-law shape and solar abundances for the absorbing material, these authors find that the observed spectra are best fit in the redshift range $3.8 \lesssim z \lesssim 4.5$ (90\% confidence interval). This falls at the high end of our allowed redshift distribution and would suggest that GRB 090709A was one of the brightest GRBs (in terms of $\left.E_{\gamma, \text { iso }}\right)$ ever detected.

Combining these results, we believe the redshift of GRB 090709A falls somewhere in the range $0.5 \lesssim z \lesssim 4$.0. The distance limits derived here are not particularly constraining. In what follows, we adopt a fiducial redshift of $\sim 1$. The implied isotropic gamma-ray energy release is then $E_{\gamma, \text { iso }} \approx 3.1 \times 10^{53} \mathrm{erg}$, and the observed host-galaxy limits correspond to $M_{i^{\prime}}(\mathrm{AB})>-18.6$ mag and $M_{K}(\mathrm{AB})>-19.5 \mathrm{mag}$. Both results are consistent with the observed long-duration GRB population (Butler et al. 2007; Savaglio et al. 2009). Where appropriate, we discuss the impact of varying the distance on the results.

Clearly the X-ray afterglow provides the best sampling, so we begin our investigation there. After the first $\sim 10^{3} \mathrm{~s}$, 
the X-ray decay is quite smooth. There is no evidence for dramatic variability on short time scales as seen, for example, in the X-ray light curve of GRB 070610 (Kasliwal et al. 2008; Stefanescu et al. 2008; Castro-Tirado et al. 2008). Most strikingly, a detailed timing analysis of X-ray afterglow of both XRT and XMM-Newton observations of GRB 090709A reveals no evidence for periodicity superposed on the overall power-law decline (Mirabal \& Gotthelf 2009; De Luca et al. 2010). Such behavior is almost always seen in the decaying X-ray phase of SGR flares, and its absence is a challenge to any magnetar model.

On the other hand, synchrotron afterglow theory predicts a series of possible relationships between the spectral and temporal indices known as closure relations. For the X-ray afterglow of GRB 090709A, the observed values $\left(\alpha_{X} \approx 1.4\right.$, $\beta_{X} \approx 1$; Section 3) agree well with theoretical predictions if the $\mathrm{X}$-ray band falls below the cooling frequency (i.e., $v_{X}<v_{c}$ ) and the shock wave is expanding into a constant-density circumburst medium $\left(\rho \propto r^{0}\right): \alpha=3 \beta / 2$. It is relatively unusual to observe the cooling frequency above the X-ray bands at this early time, though not unprecedented (e.g., GRB 060418; Cenko et al. 2010). We note, however, that the case of the $X$-ray band falling above the cooling frequency $\left(v_{X}>v_{c}\right)$ is marginally acceptable, particularly if the shallower initial decay index from the broken power-law fit $\left(\alpha_{X, 1} \approx 1.2\right.$; Section 3$)$ is used (light-curve behavior above $v_{c}$ is independent of the circumburst medium density profile). The case of the X-ray bandpass falling below $v_{c}$ in a wind-like circumburst environment $\left(\rho \propto r^{-2}\right)$ is strongly ruled out for both X-ray light-curve fits.

The observed optical spectral index $\left(\beta_{O}=3.8\right)$ is much steeper than predicted by afterglow theory. Furthermore, if we consider the last reported $K$-band detection from Calar Alto at $t \approx 0.8$ day (when the $\mathrm{X}$-ray light curve is dominated by afterglow light), the measured X-ray to optical spectral index is $\beta_{\mathrm{OX}} \approx 0.26$, well below the "dark" burst threshold $\left(\beta_{\mathrm{OX}}<0.5\right.$; Jakobsson et al. 2004). Both facts suggest a significant amount of optical extinction along the line of sight.

In order to make the intrinsic $K$-band to X-ray flux ratio consistent with predicted values $\left(\beta_{\mathrm{OX}}>0.5\right)$, the observed $K$-band flux must be suppressed by dust along the line of sight by at least $A_{K, \mathrm{obs}} \gtrsim 2 \mathrm{mag}$. However, if the synchrotron cooling frequency does indeed fall above the X-ray bandpass, the SED should be continuous from the X-rays through the optical; i.e., $\beta_{\mathrm{OX}} \approx 1$. In this case, the required host extinction would rise sharply, to $A_{K \text {,obs }} \approx 6$ mag.

We can estimate the rest-frame $V$-band extinction $\left(A_{V \text {, host }}\right)$ by assuming that the optical SED remains largely unchanged from our measurement at $t_{0}+350 \mathrm{~s}\left(\beta_{O} \approx 3.8\right)$. If the optical to X-ray spectral index is in the range $0.5 \lesssim \beta_{\text {OX }} \lesssim 1.0$, we find a hostgalaxy extinction of $A_{V \text {, host }} \approx 4-8 \mathrm{mag}\left(A_{V \text {, host }} \approx 2-6 \mathrm{mag}\right)$ for $z \approx 1(z \approx 3)$.

Though most well-studied long-duration afterglow sightlines exhibit relatively small amounts of host extinction $\left(\left\langle A_{V}\right\rangle \approx\right.$ 0.2 mag; Kann et al. 2006, 2007; Schady et al. 2010), this result is likely strongly biased toward low- $A_{V}$ sightlines (e.g., Cenko et al. 2009). GRB afterglows with host $A_{V}$ values as large as $\sim 3$ mag have been reported in the literature (e.g., Prochaska et al. 2009; Rol et al. 2007), and even larger values have been inferred for GRBs without measured redshifts (e.g., Tanvir et al. 2008; Perley et al. 2009b). Like GRB 090709A, these highly obscured events often masquerade as high-redshift candidates at first (e.g., Aoki et al. 2009; Morgan et al. 2009). Large host columns are on occasion expected given the likely origin of long-duration GRBs in giant molecular clouds in the disks of their host galaxies. Furthermore, the nondetection of the host galaxy at millimeter wavelengths (Morgan \& Bower 2009) is not particularly constraining, as most dark-burst host galaxies do not differ dramatically from the larger long-duration GRB population, suggesting a patchy dust distribution (e.g., Berger et al. 2003; Perley et al. 2009b).

Examining a variety of different extinction laws over the redshift range $0.5 \lesssim z \lesssim 4$, we find that a large selective extinction coefficient $\left(R_{V} \approx 4-15\right)$ is required to recover an intrinsic optical spectral slope of $0 \lesssim \beta_{O} \lesssim 1$ in nearly all cases. For comparison, the global value of $R_{V}$ for the Milky Way (SMC) is 3.08 (2.93; Pei 1992). A large value of $R_{V}(\sim 4)$ was inferred for the heavily obscured GRB 080607 (Prochaska et al. 2009), similar to the lines of sight toward molecular clouds in the Milky Way, and may therefore be common for heavily extinguished environments. The extinction law from Calzetti et al. (1994) can provide a reasonable fit across the entire redshift range of interest. To a large extent this is due to the featureless nature of this extinction law outside the far-ultraviolet, which by default maintains the power-law shape of the observed optical SED. On the other hand, an SMC-like extinction law is only consistent with $z \gtrsim 1.5$. Extinction laws with a strong $2175 \AA$ bump (Milky Way, LMC) also favor redshifts near our upper limit.

We have also attempted to constrain the host-galaxy column density $\left(N_{H \text {,host }}\right)$ using the late-time $\left(t \gtrsim 10^{3} \mathrm{~s}\right)$ XRT spectrum. Not surprisingly, the required column rises sharply as a function of redshift, from $N_{H \text {, host }}=(3.5 \pm 0.5) \times 10^{21} \mathrm{~cm}^{-2}$ at $z \approx 0.5$ to $N_{H \text {,host }}=(6.6 \pm 1.0) \times 10^{22} \mathrm{~cm}^{-2}$ at $z \approx 3$. Our X-ray spectral fits are consistent with those derived in De Luca et al. (2010). At the smallest distances, the inferred dust-to-metals ratio $\left(A_{V \text {,host }} / N_{H \text {,host }} \approx 1-2 \times 10^{-21}\right)$ is in excess of the Galactic value. This result is inconsistent with observations of previous GRB sightlines (e.g., Galama \& Wijers 2001; Watson et al. 2006), where the dust-to-metals ratio is typically well below the Galactic value. At larger distances, $A_{V \text {,host }}$ drops and $N_{H \text {,host }}$ rises, lowering the inferred ratio to $6-17 \times 10^{-23}$ at $z \approx 3$. This result suggests that GRB 090709A may fall at the high end of our preferred distance scale.

Assuming a Calzetti et al. (1994) extinction law with a large $R_{V}$ can account for the optical SED, we have constructed sample models for the $\mathrm{X}$-ray, $K$-band, and radio afterglow emission from GRB 090709A using the software described by Yost et al. (2003). No single forward-shock model can account for all of the observed data; a sample attempt is shown with dashed lines in Figure 6. The parameters of the dashed-line model are relatively contrived to provide an extremely small cooling frequency $\left(v_{c}<v_{O}\right.$ for $t<0.01$ day), unlike what we inferred previously based on the X-ray spectral and temporal indices. This is not entirely surprising, as we know the early X-ray emission is often dominated by other components besides the afterglow (Nousek et al. 2006). In particular, energy injection in this phase can significantly alter the subsequent evolution of the afterglow.

If we only consider the emission at $t \gtrsim 0.1$ day, when the afterglow emission from the forward shock appears to dominate the observed emission, we can easily find good models with $v_{c}>v_{X}$. One example is shown as the solid line in Figure 6. Lower redshifts typically provide better fits to the bright X-ray flux. However, the lack of optical and radio data at these times leaves the basic physical parameters of such models largely unconstrained. 


\section{CONCLUSION}

We now return again to our original question: what is the origin of GRB 090709A? A careful examination of the high-energy light curve indicates that the previously claimed detection of QPOs was in our opinion overstated: with a proper accounting for the underlying spectrum, we detect the periodic signal with only marginal $(\sim 2 \sigma)$ significance. Together with the lack of obvious environmental clues of recent star formation, it seems unlikely that GRB 090709A could be caused by an SGRlike outburst from a highly magnetized NS.

Independent of the high-energy properties, we have shown that GRB 090709A was almost certainly a cosmologically distant event. Even if associated with an older stellar population and therefore free of the requirement to be nearby recent star formation, the X-ray and optical extinction in excess of the Galactic value are difficult to reconcile with an origin in the Milky Way or nearby universe. The bright high-energy fluence and X-ray afterglow favor a more nearby event $(z \approx 1)$, while the X-ray (De Luca et al. 2010) and optical (this work) extinction properties point to a more distant origin $(z \approx 4)$. Perhaps the true value for the distance falls somewhere in between these extremes.

A long-duration GRB (and, by assumption, the core collapse of a massive star), on the other hand, can naturally account for the majority of the observed properties of GRB 090709A: the large line-of-sight extinction, the lack of an obvious host galaxy, and the late-time afterglow decay. In many ways, GRB 090709A appears to be a relatively close analog of GRB 080607 (Prochaska et al. 2009): in particular, the large host extinction $\left(A_{K \text {,obs }} \gtrsim 2 \mathrm{mag}\right)$ and the high selective extinction $\left(R_{V} \gtrsim 4\right)$ are shared by both events. Such highly obscured GRBs may be relatively common, but are more likely to be discovered recently due to the prompt localization capabilities of Swift and rapid ground-based follow-up observations in the NIR. If so, it will be important to incorporate such optically dark events into systematic studies of the GRB population to minimize observational bias (e.g., Perley et al. 2009b).

Subsequent observations could definitively resolve this issue: additional outbursts from this location would require an SGRlike (i.e., nondestructive) progenitor. On the other hand, the detection of a faint host galaxy at the location of the afterglow (and in particular measurement of a substantial redshift) would provide even stronger evidence in support of our long-duration GRB hypothesis.

We thank Eliot Quataert and Tony Piro for valuable discussions, Cullen Blake and Bethany Cobb for their assistance in automating and operating the PAIRITEL telescope, C. Álvarez for his help with the GTC observations, G. Bergond for his support during the $3.5 \mathrm{~m}$ CAHA observations, Mansi Kasliwal and Fiona Harrison for assistance in operating P60, and Andy Boden for acquiring the Keck/LGS data. S.B.C. and A.V.F. are grateful for generous support from Gary and Cynthia Bengier, the Richard and Rhoda Goldman Fund, NASA/Swift Guest Investigator grant NNX09AL08G, and National Science Foundation (NSF) grant AST0908886. A.N.M. acknowledges support from an NSF Graduate Research Fellowship. The research of J.G. and A.J.C.-T. is supported by the Spanish programs ESP2005-07714-C03-03, AYA2004-01515, AYA200767627-C03-03, AYA2008-03467/ESP, and AYA2009-14000C03-01. The National Energy Research Scientific Computing Center, which is supported by the Office of Science of the U.S.
Department of Energy under Contract No. DE-AC0205CH11231, provided staff, computational resources and data storage for this project. P.E.N., A.V.F., and J.S.B. acknowledge support from the US Department of Energy Scientific Discovery through Advanced Computing program under contract DE-FG02-06ER06-04. P60 operations are funded in part by NASA through the Swift Guest Investigator Program (grant no. NNG06GH61G). This publication has made use of data obtained from the Swift interface of the High-Energy Astrophysics Archive (HEASARC), provided by NASA's Goddard Space Flight Center. PAIRITEL is operated by the Smithsonian Astrophysical Observatory (SAO) and was made possible by a grant from the Harvard University Milton Fund, a camera loan from the University of Virginia, and continued support of the SAO and UC Berkeley. The PAIRITEL project and those working on PAIRITEL data are further supported by NASA/Swift Guest Investigator grants NNG06GH50G and NNX08AN84G. Based on observations made with the Gran Telescopio Canarias (GTC), installed in the Spanish Observatorio del Roque de los Muchachos of the Instituto de Astrofisica de Canarias, in the island of La Palma. Based in part on observations collected at the Centro Astronómico Hispano Alemán (CAHA) at Calar Alto, operated jointly by the Max-Planck Institut für Astronomie and the Instituto de Astrofísica de Andalucía (CSIC). We thank Calar Alto Observatory for allocation of Director's Discretionary Time. Some of the data presented herein were obtained at the W. M. Keck Observatory, which is operated as a scientific partnership among the California Institute of Technology, the University of California, and NASA; the Observatory was made possible by the generous financial support of the W. M. Keck Foundation. The authors recognize and acknowledge the very significant cultural role and reverence that the summit of Mauna Kea has always had within the indigenous Hawaiian community; we are most fortunate to have the opportunity to conduct observations from this mountain.

Facilities: Swift (BAT; XRT), FLWO:1.2m (PAIRITEL), PO:1.2m (QUEST), PO:1.5m, CAO:3.5m (OMEGA 2000$)$, GTC (OSIRIS), Keck:II (NIRC2/LGS), VLA

\section{REFERENCES}

Aoki, K., Ishii, M., Kuzuhara, M., Takahashi, Y., \& Kawai, N. 2009, GCN Circ., 9634, 1

Barthelmy, S. D., et al. 2005a, Space Sci. Rev., 120, 143

Barthelmy, S. D., et al. 2005b, Nature, 438, 994

Beloborodov, A. M., Stern, B. E., \& Svensson, R. 1998, ApJ, 508, L25

Beloborodov, A. M., Stern, B. E., \& Svensson, R. 2000, ApJ, 535, 158

Berger, E., Cowie, L. L., Kulkarni, S. R., Frail, D. A., Aussel, H., \& Barger, A. J. 2003, ApJ, 588, 99

Berger, E., et al. 2005, Nature, 438, 988

Bloom, J. S., Kulkarni, S. R., \& Djorgovski, S. G. 2002, AJ, 123, 111

Bloom, J. S., Starr, D. L., Blake, C. H., Skrutskie, M. F., \& Falco, E. E. 2006a, in ASP Conf. Ser. 351, Astronomical Data Analysis Software and Systems XV, ed. C. Gabriel, C. Arviset, D. Ponz, \& E. Solano (San Francisco, CA: ASP), 751

Bloom, J. S., et al. 2006b, ApJ, 638, 354

Bloom, J. S., et al. 2009, ApJ, 691, 723

Burrows, D. N., et al. 2005, Space Sci. Rev., 120, 165

Butler, N. R. 2007, AJ, 133, 1027

Butler, N. R., Bloom, J. S., \& Poznanski, D. 2010, ApJ, 711, 495

Butler, N. R., \& Kocevski, D. 2007, ApJ, 663, 407

Butler, N. R., Kocevski, D., Bloom, J. S., \& Curtis, J. L. 2007, ApJ, 671, 656

Calzetti, D., Kinney, A. L., \& Storchi-Bergmann, T. 1994, ApJ, 429, 582

Cameron, P. B., et al. 2005, Nature, 434, 1112

Canal, R., \& Schatzman, E. 1976, A\&A, 46, 229

Castro-Tirado, A. J., et al. 2005, A\&A, 439, L15

Castro-Tirado, A. J., et al. 2008, Nature, 455, 506 
Cenko, S. B., et al. 2006, PASP, 118, 1396

Cenko, S. B., et al. 2008, ApJ, 677, 441

Cenko, S. B., et al. 2009, ApJ, 693, 1484

Cenko, S. B., et al. 2010, ApJ, 711, 641

Chandra, P., \& Frail, D. A. 2009, GCN Circ., 9657, 1

Chapman, R., Priddey, R. S., \& Tanvir, N. R. 2009, MNRAS, 395, 1515

Cheng, K. S., \& Wang, X. Y. 2003, ApJ, 593, L85

Christensen, L., Hjorth, J., \& Gorosabel, J. 2004, A\&A, 425, 913

Corbel, S., \& Eikenberry, S. S. 2004, A\&A, 419, 191

Della Valle, M. D., et al. 2006, Nature, 444, 1050

De Luca, A., Esposito, P., Israel, G. L., Götz, D., Novara, G., Tiengo, A., \& Mereghetti, S. 2010, MNRAS, 402, 1870

Djorgovski, S. G., et al. 2008, Astron. Nachr., 329, 263

Duncan, R. C., \& Thompson, C. 1992, ApJ, 392, L9

Eichler, D., Livio, M., Piran, T., \& Schramm, D. N. 1989, Nature, 340, 126

Fahlman, G. G., \& Gregory, P. C. 1981, Nature, 293, 202

Feroci, M., Frontera, F., Costa, E., Amati, L., Tavani, M., Rapisarda, M., \& Orlandini, M. 1999, ApJ, 515, L9

Fishman, G. J., et al. 1994, Science, 264, 1313

Frail, D. A., Kulkarni, S. R., \& Bloom, J. S. 1999, Nature, 398, 127

Fruchter, A. S., et al. 2006, Nature, 441, 463

Fynbo, J. P. U., et al. 2006, Nature, 444, 1047

Gaensler, B. M., Gotthelf, E. V., \& Vasisht, G. 1999, ApJ, 526, L37

Gaensler, B. M., et al. 2005, Nature, 434, 1104

Galama, T. J., \& Wijers, R. A. M. J. 2001, ApJ, 549, L209

Gal-Yam, A., et al. 2006, Nature, 444, 1053

Gehrels, N., et al. 2004, ApJ, 611, 1005

Gehrels, N., et al. 2005, Nature, 437, 851

Gehrels, N., et al. 2006, Nature, 444, 1044

Gelfand, J. D., \& Gaensler, B. M. 2007, ApJ, 667, 1111

Golenetskii, S., Aptekar, R., Mazets, E., Pal'Shin, V., Frederiks, D., Oleynik, P., Ulanov, M., \& Svinkin, D. 2009, GCN Circ., 9647

Gorosabel, J., et al. 2006, A\&A, 450, 87

Gotz, D., Mereghetti, S., von Kienlin, A., \& Beck, M. 2009, GCN Circ., 9649

Grupe, D., Nousek, J. A., Berk, D. E. V., Roming, P. W. A., Burrows, D. N., Godet, O., Osborne, J., \& Gehrels, N. 2007, AJ, 133, 2216

Guidorzi, C., et al. 2009, GCN Circ., 9648

Hjorth, J., et al. 2005, ApJ, 630, L117

Hurley, K., Briggs, M. S., Kippen, R. M., Kouveliotou, C., Meegan, C., Fishman, G., Cline, T., \& Boer, M. 1999a, ApJS, 120, 399

Hurley, K., et al. 1999b, Nature, 397, 41

Hurley, K., et al. 2005, Nature, 434, 1098

Hurley, K., et al. 2010, MNRAS, 403, 342

Jakobsson, P., Hjorth, J., Fynbo, J. P. U., Watson, D., Pedersen, K., Björnsson, G., \& Gorosabel, J. 2004, ApJ, 617, L21

Jordi, K., Grebel, E. K., \& Ammon, K. 2006, A\&A, 460, 339

Kalberla, P. M. W., Burton, W. B., Hartmann, D., Arnal, E. M., Bajaja, E., Morras, R., \& Pöppel, W. G. L. 2005, A\&A, 440, 775

Kann, D. A., Klose, S., \& Zeh, A. 2006, ApJ, 641, 993

Kann, D. A., et al. 2007, arXiv:0712.2186

Kasliwal, M. M., et al. 2008, ApJ, 678, 1127

Klose, S., et al. 2004, ApJ, 609, L13

Kouveliotou, C., Meegan, C. A., Fishman, G. J., Bhat, N. P., Briggs, M. S., Koshut, T. M., Paciesas, W. S., \& Pendleton, G. N. 1993, ApJ, 413, L101

Kouveliotou, C., et al. 1998, Nature, 393, 235

Kulkarni, S. R., Kaplan, D. L., Marshall, H. L., Frail, D. A., Murakami, T., \& Yonetoku, D. 2003, ApJ, 585, 948

Lamb, R. C., Fox, D. W., Macomb, D. J., \& Prince, T. A. 2002, ApJ, 574, L29

Laros, J. G., et al. 1997, ApJS, 110, 157

Malesani, D., Telting, J., Wilson, P., Jakobsson, P., Fynbo, J. P. U., Leloudas, G., Levan, A. J., \& Wiersema, K. 2009, GCN Circ., 9644

Markwardt, C. B., Gavriil, F. P., Palmer, D. M., Baumgartner, W. H., \& Barthelmy, S. D. 2009, GCN Circ., 9645
Mazets, E. P., Cline, T. L., Aptekar', R. L., Butterworth, P. S., Frederiks, D. D. Golenetskii, S. V., Il'Inskii, V. N., \& Pal'Shin, V. D. 1999, Astron. Lett., 25, 635

Mazets, E. P., Golenetskii, S. V., Gurian, I. A., \& Ilinskii, V. N. 1982, Ap\&SS, 84, 173

Mazets, E. P., Golentskii, S. V., Ilinskii, V. N., Aptekar, R. L., \& Guryan, I. A. 1979, Nature, 282, 587

Mazets, E. P., et al. 2008, ApJ, 680, 545

Mereghetti, S. 2008, A\&AR, 15, 225

Mereghetti, S., et al. 2009, arXiv:0908.0414

Mirabal, N., \& Gotthelf, E. V. 2009, GCN Circ., 9696, 1

Monet, D. G., et al. 2003, AJ, 125, 984

Morgan, A. N., Bloom, J. S., \& Klein, C. R. 2009, GCN Circ., 9635

Morgan, A. N., \& Bower, G. C. 2009, GCN Circ., 9685

Morris, D. C., et al. 2009, GCN Circ., 9625

Muno, M. P., et al. 2006, ApJ, 636, L41

Narayan, R., Paczynski, B., \& Piran, T. 1992, ApJ, 395, L83

Nousek, J. A., et al. 2006, ApJ, 642, 389

Ofek, E. O. 2007, ApJ, 659, 339

Ofek, E. O., et al. 2006, ApJ, 652, 507

Ofek, E. O., et al. 2007, ApJ, 662, 1129

Ofek, E. O., et al. 2008, ApJ, 681, 1464

Ohno, M., et al. 2009, GCN Circ., 9653

Oke, J. B., \& Gunn, J. E. 1983, ApJ, 266, 713

Osborne, J. P., Beardmore, A. P., Evans, P. A., \& Goad, M. R. 2009, GCN Circ., 9636, 1

Pei, Y. C. 1992, ApJ, 395, 130

Perley, D. A., et al. 2009a, ApJ, 696, 1871

Perley, D. A., et al. 2009b, AJ, 138, 1690

Piran, T. 2005, Rev. Mod. Phys., 76, 1143

Prochaska, J. X., et al. 2009, ApJ, 691, L27

Rol, E., et al. 2007, ApJ, 669, 1098

Rowlinson, A., \& Morris, D. C. 2009, GCN Circ., 9642

Sakamoto, T., et al. 2009, GCN Circ., 9640

Salvaterra, R., et al. 2009, Nature, 461, 1258

Sari, R., Piran, T., \& Narayan, R. 1998, ApJ, 497, L17

Savaglio, S., Glazebrook, K., \& LeBorgne, D. 2009, ApJ, 691, 182

Schady, P., et al. 2010, MNRAS, 401, 2773

Schlegel, D. J., Finkbeiner, D. P., \& Davis, M. 1998, ApJ, 500, 525

Skrutskie, M. F., et al. 2006, AJ, 131, 1163

Stanek, K. Z., et al. 2006, Acta Astron., 56, 333

Stefanescu, A., Kanbach, G., Słowikowska, A., Greiner, J., McBreen, S., \& Sala, G. 2008, Nature, 455, 503

Suzuki, M., Morikawa, M., \& Joichi, I. 2002, in ESO Astrophysics Symp., Lighthouses of the Universe: The Most Luminous Celestial Objects and Their Use for Cosmology: Proc. MPA/ESO/MPE/USM Joint Astronomy Conference, ed. M. Gilfanov, R. Sunyaev, \& E. Churazov (Berlin: Springer)

Tanvir, N. R., Chapman, R., Levan, A. J., \& Priddey, R. S. 2005, Nature, 438, 991

Tanvir, N. R., et al. 2008, MNRAS, 388, 1743

Tanvir, N. R., et al. 2009, Nature, 461, 1254

van der Horst, A. J., et al. 2009, GCN Circ., 9499

Vasisht, G., \& Gotthelf, E. V. 1997, ApJ, 486, L129

Vaughan, S. 2005, A\&A, 431, 391

Voges, W., et al. 1999, A\&A, 349, 389

Vrba, F. J., Henden, A. A., Luginbuhl, C. B., Guetter, H. H., Hartmann, D. H., \& Klose, S. 2000, ApJ, 533, L17

Wainwright, C., Berger, E., \& Penprase, B. E. 2007, ApJ, 657, 367

Watson, D., et al. 2006, ApJ, 652, 1011

Wizinowich, P. L., et al. 2006, PASP, 118, 297

Woods, P. M., \& Thompson, C. 2006, in Cambridge Astrophysics Series 39, Compact Stellar X-ray sources, ed. W. Lewin \& M. van der Klis (Cambridge: Cambridge Univ. Press), 547

Woosley, S. E., \& Bloom, J. S. 2006, ARA\&A, 44, 507

Yost, S. A., Harrison, F. A., Sari, R., \& Frail, D. A. 2003, ApJ, 597, 459 